\title{
Incidence and risk factors of chronic thromboembolic pulmonary hypertension after acute pulmonary embolism: a systematic review and meta-analysis of cohort studies
}

\author{
Meng Zhang ${ }^{1,2}$, Ning Wang ${ }^{3}$, Zhenguo Zhai ${ }^{4,5}$, Mingyuan Zhang ${ }^{1}$, Rongrong Zhou ${ }^{1}$, Yanyan Liu ${ }^{1}$, \\ Yuanhua Yang ${ }^{1}$ \\ ${ }^{1}$ Beijing Institute of Respiratory Medicine, Beijing Chao-Yang Hospital, Capital Medical University, Beijing 100043 , China; ${ }^{2}$ Peking University \\ Health Science Center, Beijing 100191, China; ${ }^{3}$ Department of Respiratory Medicine, Harrison International Peace Hospital, Hengshui 053000, \\ China; ${ }^{4}$ Department of Respiratory and Critical Care Medicine, China-Japan Friendship Hospital, Beijing 100029, China; ${ }^{5}$ Department of \\ Respiratory Medicine, Capital Medical University, Beijing 100069, China \\ Contributions: (I) Conception and design: M Zhang, Z Zhai, Y Yang; (II) Administrative support: None; (III) Provision of study materials or patients: \\ None; (IV) Collection and assembly of data: M Zhang, M Zhang, R Zhou; (V) Data analysis and interpretation: N Wang, Y Liu; (VI) Manuscript \\ writing: All authors; (VII) Final approval of manuscript: All authors. \\ Correspondence to: Yuanhua Yang, MD, PhD. Beijing Institute of Respiratory Medicine, Beijing Chao-Yang Hospital, Capital Medical University, No. \\ 8 Gongtinanlu, Chao-Yang District, Beijing 100043, China. Email: yyh1031@sina.com.
}

Background: The incidence and risk factors of chronic thromboembolic pulmonary hypertension $(\mathrm{CTEPH})$ after acute pulmonary embolism (PE) were still controversial. A systematic review and metaanalysis was conducted to assess the incidence and risk factors of CTEPH after acute PE.

Methods: Embase, Medline, China National Knowledge Infrastructure, Wanfang databases, and various reference lists were searched to identify studies published up to May 2018. Only cohort studies that used right heart catheterization for CTEPH diagnosis were included. The study quality was assessed using the Newcastle-Ottawa Scale (NOS). All analyses were conducted with the meta package in R software (3.2.2).

Results: Fifteen studies met the inclusion criteria. The overall incidence of CTEPH after acute PE, with a median follow-up from 6 to 94.3 months, was 3.13\% (95\% CI: 2.11-4.63\%). The incidence of studies from China [4.46\% (95\% CI: 1.68-11.32\%)] was slightly higher than from Europe [2.82\% (95\% CI: 1.82-4.34\%)]. However, there was no significant difference between these two groups $(\mathrm{P}=0.39)$. Subgroup analyses of confirmed diagnostic method showed that compared studies using right heart catheterization [3.25\% (95\% CI: $2.12-4.97 \%)$ ], studies using right heart catheterization combined with bundled tests tended to yield a similar data [2.40\% (95\% CI: 0.97-5.81\%), P=0.54]. Previous/recurrent PE or previous VTE, idiopathic PE and right heart dysfunction were considered as risk factors of CTEPH in patients with a previous PE.

Conclusions: CTEPH is not a rare complication of acute PE. Close follow-up and implementation of a comprehensive screening program are important, especially in patients with independent risk factors.

Keywords: Chronic thromboembolic pulmonary hypertension (CTEPH); pulmonary embolism (PE); incidence; meta-analysis

Submitted Dec 07, 2017. Accepted for publication Jul 12, 2018.

doi: $10.21037 /$ jtd.2018.07.106

View this article at: http://dx.doi.org/10.21037/jtd.2018.07.106

(c) Journal of Thoracic Disease. All rights reserved. 


\section{Introduction}

Chronic thromboembolic pulmonary hypertension (CTEPH) is a life-threatening condition with a considerable morbidity and mortality. It is commonly believed that CTEPH is the result of a long-term complication of pulmonary embolism (PE) which is characterized by the absence of thrombus resolution due to one or more episodes of acute pulmonary embolic events. The actual incidence of CTEPH after an acute PE is still controversial. The incidence of CTEPH has been reported to occur in $4.8 \%$ patients with a previous PE in a recent study (1). Another prospective study from Italy in 2004 showed a lower estimate; around $3.8 \%$ after 2 years following acute PE (2). According to previous reports, the incidence of CTEPH had been estimated to vary from $0.4 \%$ to $14.7 \%$ in patients who survive an acute PE $(3,4)$.

To date, potential risk factors regarding CTEPH in patients with previous $\mathrm{PE}$ also remain unclear. The risk factors reported in previous studies were highly variable. Younger or older age, idiopathic PE, Previous/recurrent $\mathrm{PE}$ or previous venous thromboembolism (VTE), and a higher level of pulmonary artery systole pressure (sPAP) at the presentation were identified as the major risk factors for СТЕРН $(1,2,5)$.

In order to better understand the incidence and risk factors of CTEPH in patients with a previous PE, we conducted a systematic review and meta-analysis of the literature.

\section{Methods}

Embase, Medline, China National Knowledge Infrastructure, Wanfang database, and various reference lists were searched to identify studies published up to May 2018. A comprehensive literature search of Embase, Medline, China National Knowledge Infrastructure and Wanfang databases was conducted to identify all studies published up to May 2018. We also manually searched relevant articles from the reference list. The search terms included keywords for CTEPH ("chronic thromboembolic pulmonary hypertension" or "CTEPH") and the outcomes of interest ("incidence", "prevalence", "epidemiology", "morbidity", "predictor", "risk factor”, or "prognosis"). The reporting of this meta-analysis and systematic review is conducted in accordance with the Preferred Reporting Items for Systematic Reviews and Meta-Analyses (PRISMA) statement (6).
Studies that met the following criteria were included: (I) original full articles written in English or Chinese; (II) cohort studies on reporting the incidence or risk factors of CTEPH in patients with acute PE; (III) enrolled patients $\geq 18$ years old; (IV) enrolled patients with PE diagnosed based on computed tomographic pulmonary angiography (CTPA) or ventilation-perfusion scintigraphy (V/Q scan); (V) the diagnosis of CTEPH was confirmed in patients with pre-capillary $\mathrm{PH}$ defined as mean pulmonary artery pressure $\geq 25 \mathrm{mmHg}$ and pulmonary capillary wedge pressure $\leq 15 \mathrm{mmHg}$ through the right heart catheterization and at least one segmental perfusion defect on CTPA or V/Q scan; (VI) studies with a minimum follow-up of half a year; (VII) the most recent study with the most integral data would be included, if more than one study had been published using the same cohorts.

We excluded studies as follows: (I) abstracts, reviews, and conference proceedings that were not considered eligible; (II) studies with insufficient data for calculation of incidence; (III) low quality studies assessed by the criteria of the Newcastle-Ottawa Scale (NOS).

Data extraction was independently conducted by two investigators with disagreements resolved through discussion, which included the following information: the first author's name, year of publication, sample size and characteristics, study type, follow-up strategy, screening methods of CTEPH, incidence of CTEPH and risk factors related to CTEPH.

The study quality was assessed using the NOS in which the score ranged from 0 to 9 (7). Criteria for the NOS include defined inclusion criteria, diagnostic criteria, quality control, statistical analysis, sample size and its representativeness. Studies with a score $>5$ were thought to be high-quality studies. Studies with a score $\leq 5$ were thought to be low-quality studies and not included in this systematic review and meta-analysis.

The incidences of CTEPH in patients with a previous $\mathrm{PE}$ from various studies were pooled in a meta-analysis. If incidence was not reported, we extracted the incident case numbers and the enrolled patient numbers from the original data. As low incidence of CTEPH, after an acute $\mathrm{PE}$ episode, was reported in several articles, the data was transformed into logit proportion. Restricted to a small number of risk factor studies, we didn't pool the data into a meta-analysis. A systematic review of these studies was conducted to identify potential variables.

Heterogeneity was assessed using the Cochran's Q test and $\mathrm{I}^{2}$ statistics. Based on the $\mathrm{Q}$ test, a random effect model 


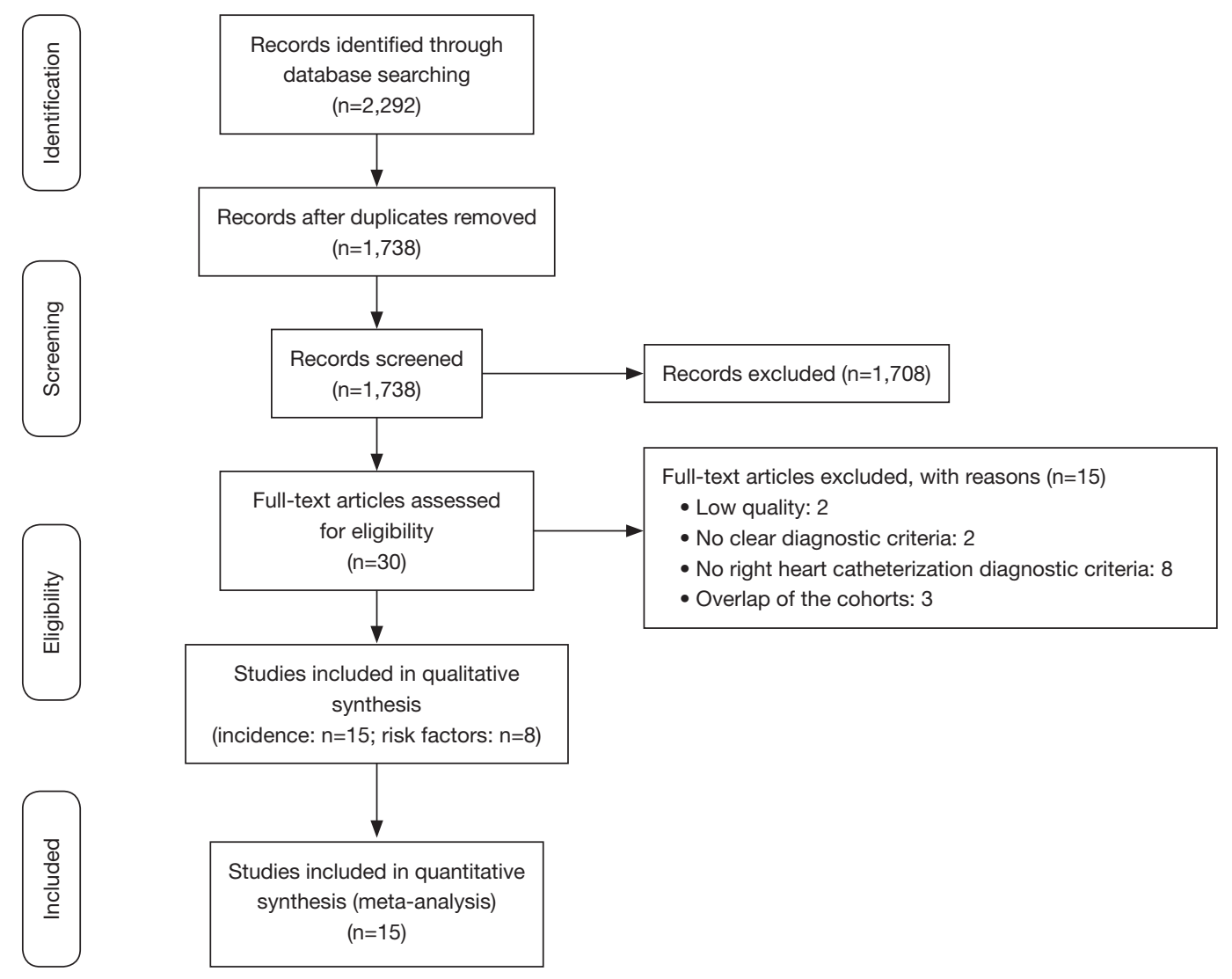

Figure 1 PRISMA flow diagram of included studies. PRISMA, Preferred Reporting Items for Systematic Reviews and Meta-Analyses.

was utilized when $\mathrm{P}$ values $<0.1$, otherwise a fixed effect model was selected. $\mathrm{I}^{2}$ statistic were utilized to measure the extent of the overall variation due to the heterogeneity; $25 \%, 50 \%$ and $75 \%$ of $\mathrm{I}^{2}$ statistic were represented as low, medium, and high heterogeneity, respectively. When substantial heterogeneity was observed $\left(\mathrm{I}^{2}>50 \%\right)$, an investigation of the sources of heterogeneity was conducted using sensitivity and subgroup analyses. During the sensitivity analyses, we excluded each study one by one and recalculated the pooled data to assess whether any one study had a dominant effect on the pooled results. A funnel plot was made to qualitatively demonstrate the publication bias in the studies. Publication bias was quantitatively assessed by the Egger test $(\mathrm{P}<0.05$ was considered statistically significant). All analyses were conducted with the meta package in $\mathrm{R}$ software (3.2.2).

\section{Results}

The literature searches yielded 2,292 records. After screening titles and abstracts, 30 full-text articles were assessed for eligibility; 15 articles were excluded after a full review: 2 articles had low quality scores $(8,9) ; 2$ articles lacked clear diagnostic criteria; 8 articles lacked right heart catheterization diagnostic criteria; 3 studies had some overlap in the study samples as included patients from Leiden University Medical Center (5,10,11); and 2 studies were based on the same cohort $(12,13)$. We avoided duplicate inclusion of data by selecting only the most complete and more recent article from each cohort $(5,13)$. From a total of 15 studies included in the final analyses (1-3,5,13-23), eight studies reported risk factors of CTEPH among the patients with a previous PE $(1,2,5$, 14-16,21,23) (Figure 1).

The characteristics of included studies are summarized in Table 1. The studies were published between 2004 and 2018. Sample sizes varied from 110 to 772 . Mean age ranged from 56 to 65 years old. Twelve studies were from Europe, and 3 studies were from China.

As shown in Figure 2, in a meta-analysis of 15 independent 


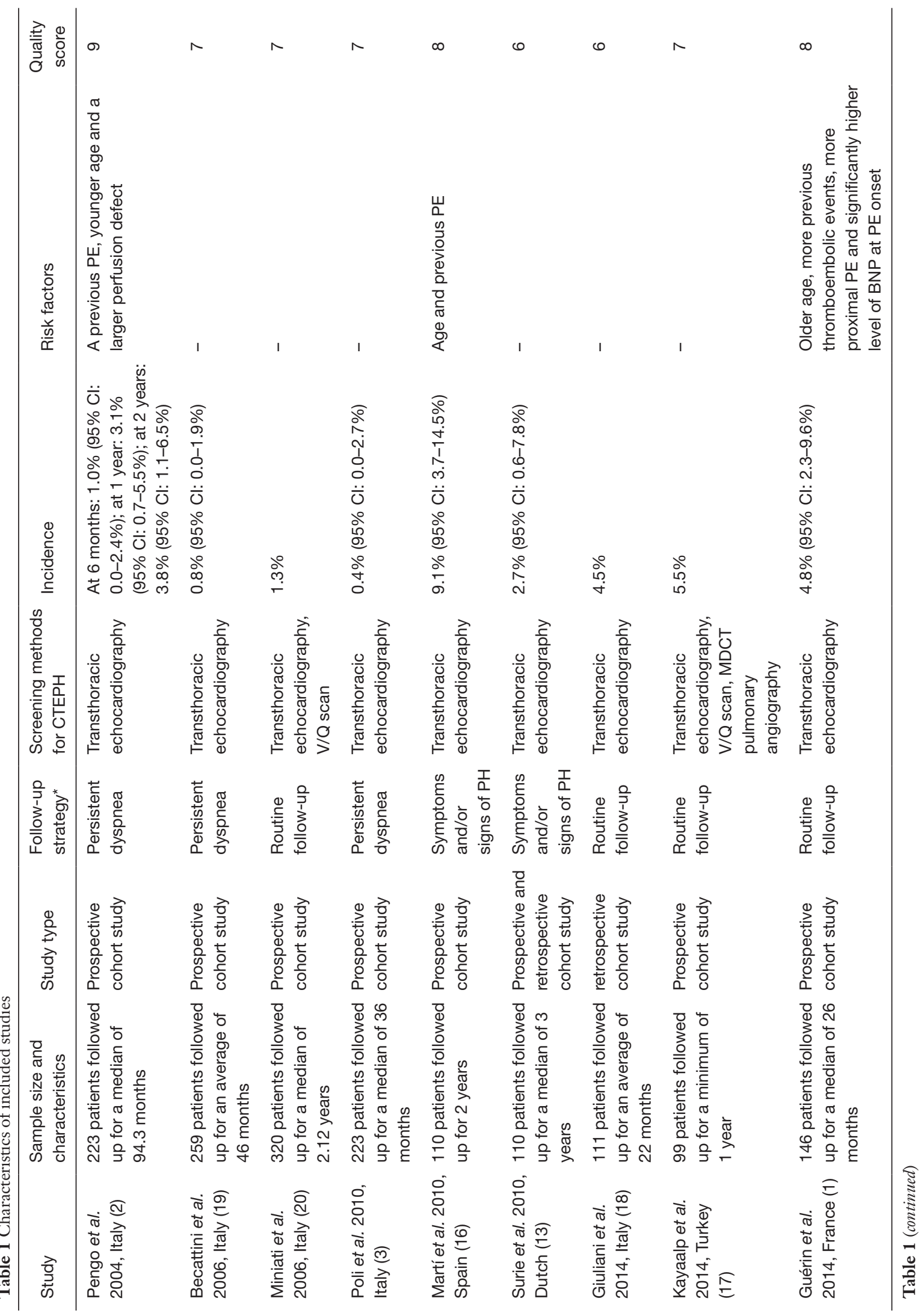




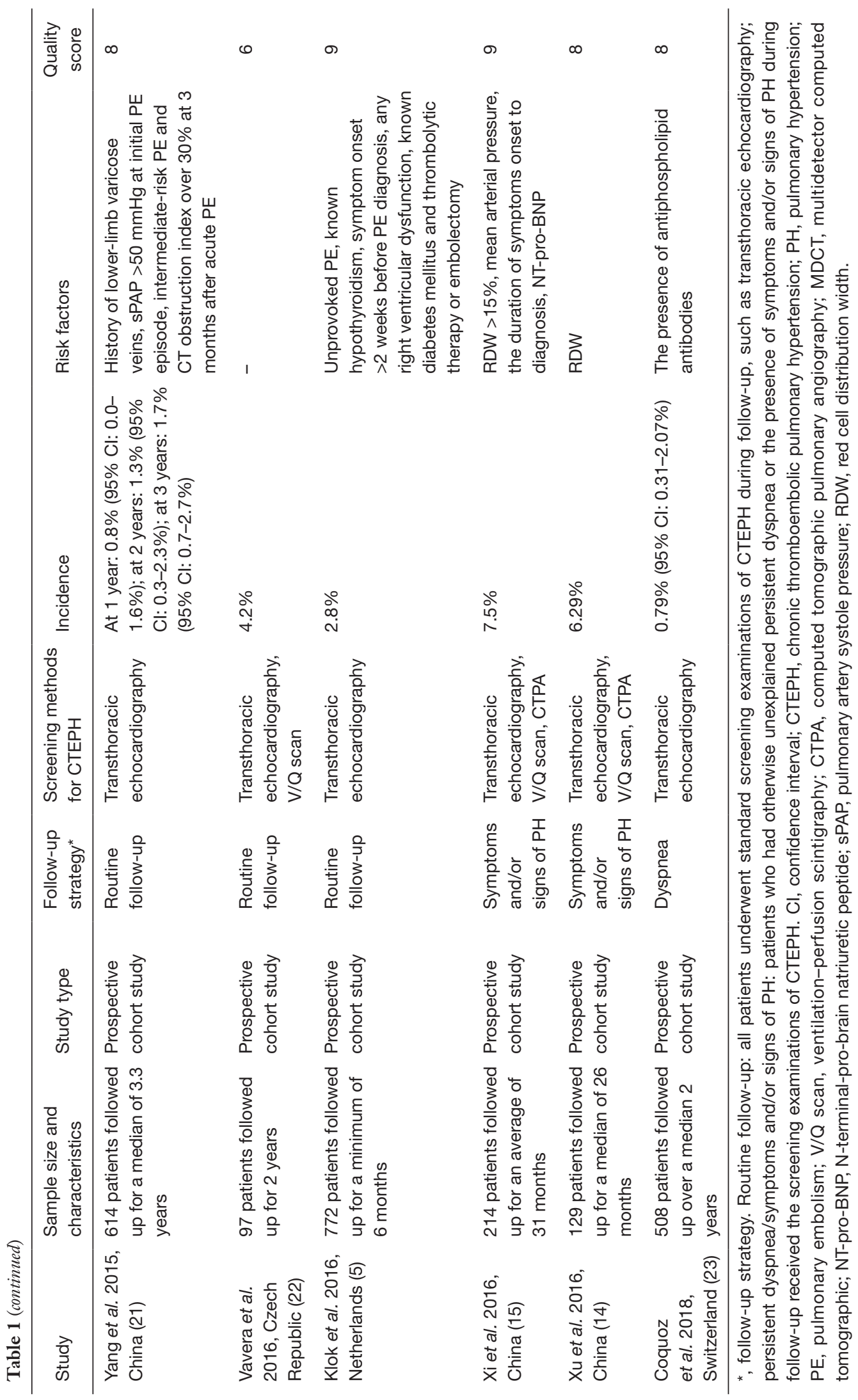




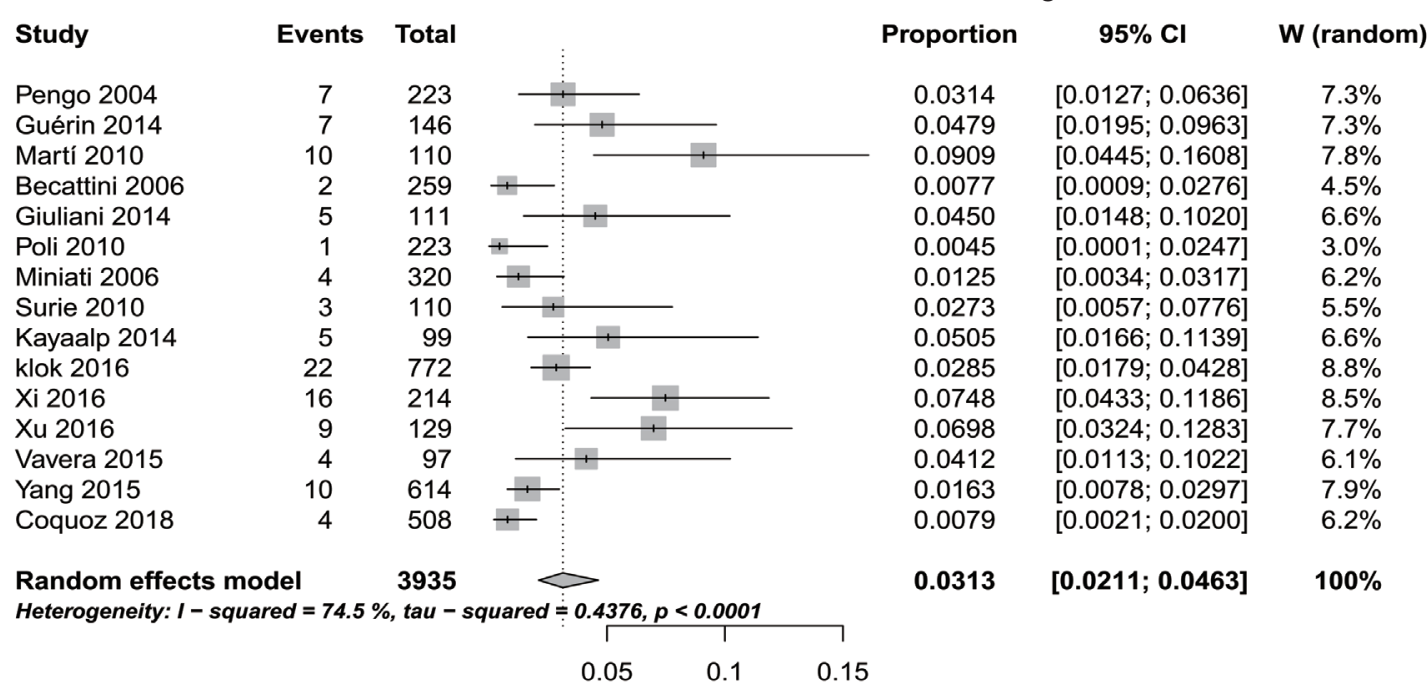

Figure 2 Meta-analysis of the incidences of CTEPH after acute PE. CTEPH, chronic thromboembolic pulmonary hypertension; PE, pulmonary embolism.

\begin{tabular}{|c|c|c|c|c|c|c|c|}
\hline \multicolumn{8}{|l|}{ Subgroup: Europe } \\
\hline Pengo 2004 & 7 & 223 & 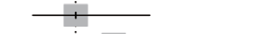 & & 0.0314 & {$[0.0127 ; 0.0636]$} & $7.3 \%$ \\
\hline Guérin 2014 & 7 & 146 & 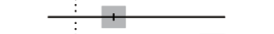 & & 0.0479 & {$[0.0195 ; 0.0963]$} & $7.3 \%$ \\
\hline Martí 2010 & 10 & 110 & $\longrightarrow$ & & 0.0909 & {$[0.0445 ; 0.1608]$} & $7.8 \%$ \\
\hline Becattini 2006 & 2 & 259 & $\mp$ & & 0.0077 & {$[0.0009 ; 0.0276]$} & $4.5 \%$ \\
\hline Giuliani 2014 & 5 & 111 & $\begin{array}{c}1 \\
1\end{array}$ & & 0.0450 & {$[0.0148 ; 0.1020]$} & $6.6 \%$ \\
\hline Poli 2010 & 1 & 223 & $\mp$ & & 0.0045 & {$[0.0001 ; 0.0247]$} & $3.0 \%$ \\
\hline Miniati 2006 & 4 & 320 & $+\vdots$ & & 0.0125 & {$[0.0034 ; 0.0317]$} & $6.2 \%$ \\
\hline Surie 2010 & 3 & 110 & 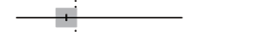 & & 0.0273 & {$[0.0057 ; 0.0776]$} & $5.5 \%$ \\
\hline Kayaalp 2014 & 5 & 99 & $\begin{array}{lll}-1 & 1 \\
& 1\end{array}$ & & 0.0505 & {$[0.0166 ; 0.1139]$} & $6.6 \%$ \\
\hline klok 2016 & 22 & 772 & 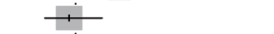 & & 0.0285 & {$[0.0179 ; 0.0428]$} & $8.8 \%$ \\
\hline Vavera 2015 & 4 & 97 & 1 & & 0.0412 & {$[0.0113 ; 0.1022]$} & $6.1 \%$ \\
\hline Coquoz 2018 & 4 & 508 & $\mp \quad$ & & 0.0079 & {$[0.0021 ; 0.0200]$} & $6.2 \%$ \\
\hline Random effects model & & 2978 & 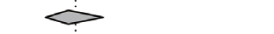 & & 0.0282 & {$[0.0182 ; 0.0434]$} & $75.9 \%$ \\
\hline \multicolumn{8}{|c|}{ Heterogeneity: $I-$ squared $=68.1 \%$, tau - squared $0.3864, p=0.0003$} \\
\hline \multicolumn{8}{|l|}{ Subgroup: China } \\
\hline Xi 2016 & 16 & 214 & + & & 0.0748 & {$[0.0433 ; 0.1186]$} & $8.5 \%$ \\
\hline Xu 2016 & 9 & 129 & 1 & & 0.0698 & {$[0.0324 ; 0.1283]$} & $7.7 \%$ \\
\hline Yang 2015 & 10 & 614 & 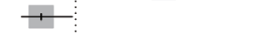 & & 0.0163 & {$[0.0078 ; 0.0297]$} & $7.9 \%$ \\
\hline Random effects model & & 957 & i & & 0.0446 & {$[0.0168 ; 0.1132]$} & $24.1 \%$ \\
\hline \multicolumn{8}{|c|}{ Heterogeneity: $I-$ squared $=88.1 \%$, tau - squared $0.6942, p=0.0002$} \\
\hline \multirow{3}{*}{\multicolumn{8}{|c|}{ Heterogeneity: $I-$ squared $=74.5 \%$, tau - squared $=0.4376, p<0.0001$}} \\
\hline & & & & & & & \\
\hline & & & $0.05 \quad 0.1$ & 0.15 & & & \\
\hline
\end{tabular}

Figure 3 Subgroup analyses of study locations.

studies, the pooled incidence of CTEPH was $3.13 \%$ (95\% CI: $2.11-4.63 \%$ ) with rates in individual studies varying from $0.4 \%$ to $9.1 \%$. The highest incidence was in Spain (16), and the lowest incidence was in Italy (5). There was a substantial amount of heterogeneity among the studies $\left(\mathrm{I}^{2}=74.5 \%, \mathrm{P}<0.0001\right.$; Figure 2). To explore the sources of heterogeneity further, we conducted subgroup analyses across key study characteristics. However, subgroup analyses didn't provide clear sources of heterogeneity. The subgroup analyses reported here provide additional insights. The incidence of CTEPH after acute PE was 4.46\% (95\% CI: $1.68-11.32 \%)$ in the Chinese population $(14,15,21)$, higher than the remaining 12 studies from Europe [2.82\% $(95 \%$ CI: 1.82-4.34\%), $\mathrm{P}=0.39$ ] (1-3,5,13,16-20,22,23) (Figure 3). Compared studies using right heart catheterization for confirmed diagnosis [3.25\% (95\% CI: 2.12-4.97\%)], 


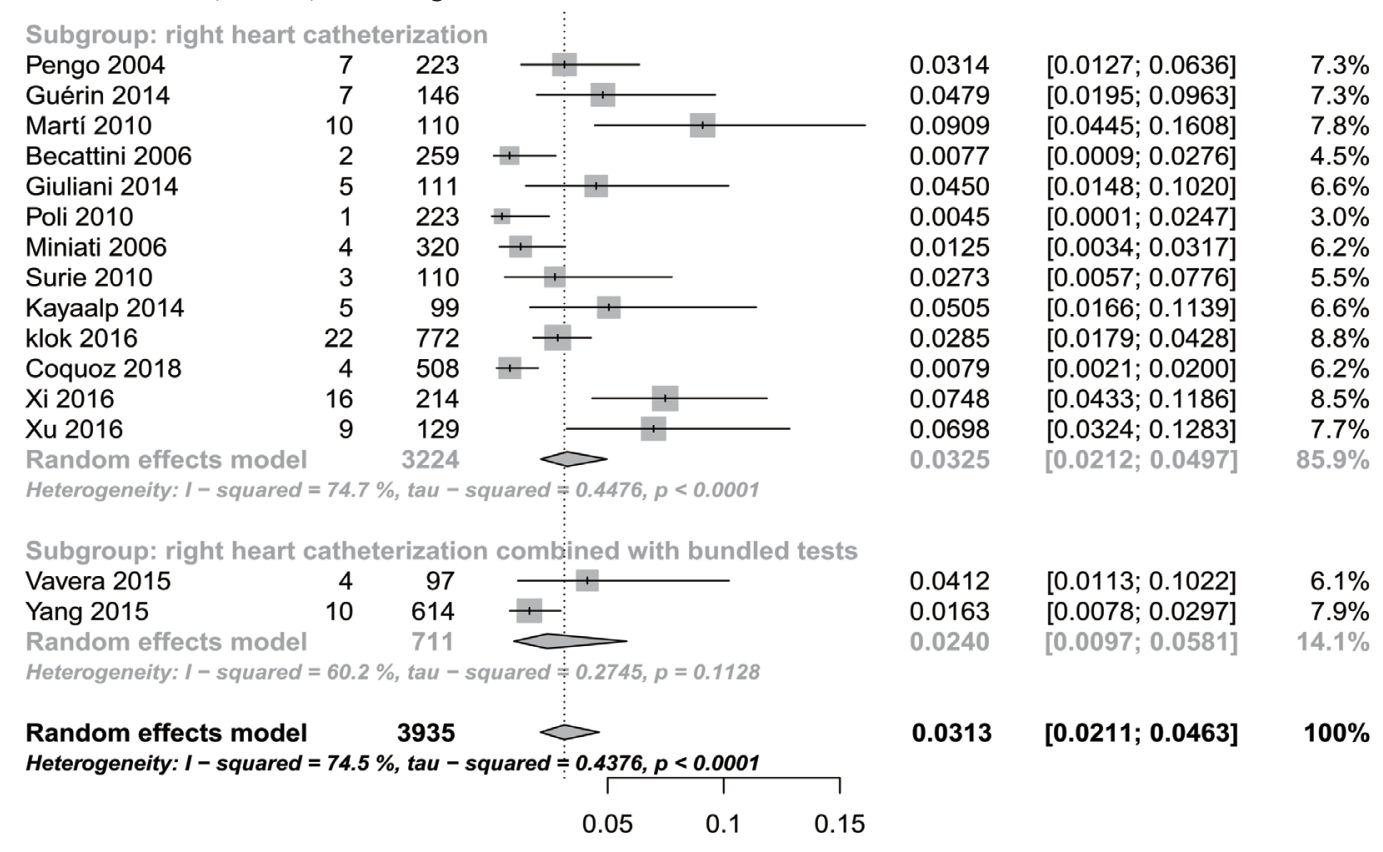

Figure 4 Subgroup analyses of confirmed diagnostic methods.

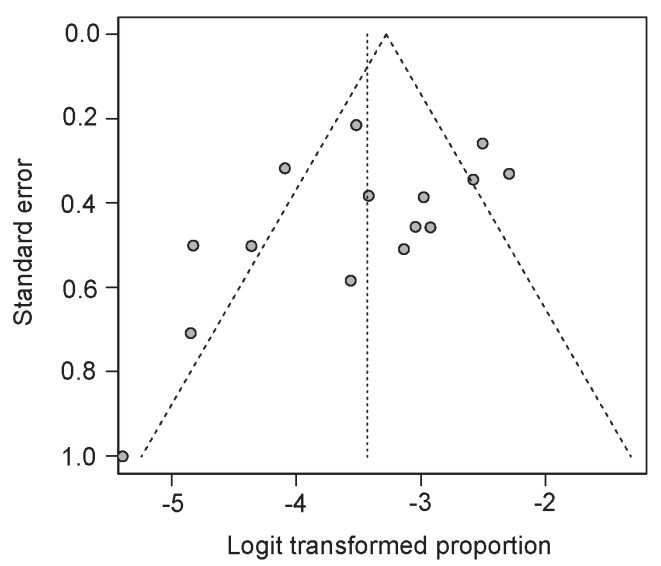

Figure 5 Funnel plot of included studies for incidence.

studies using right heart catheterization combined with bundled tests (an echocardiography and V/Q scan/CTPA along with clinical features) tended to yield a similar data [2.40\% (95\% CI: 0.97-5.81\%), P=0.54] (Figure 4). During the sensitivity analyses, we excluded studies one by one and recalculated the pooled incidence, which was close to $3.13 \%$ (95\% CI: $2.11-4.63 \%$ ), varied from $2.90 \%$ to $3.47 \%$, with no single study significantly affecting the heterogeneity.

Although the Egger test was not statistically significant $(\mathrm{P}=0.12)$, visual inspection of the funnel plot indicated asymmetry (Figure 5). By using the trim-and-fill method, which imputes hypothetical negative unpublished studies, the imputed studies produce a symmetrical funnel plot. Two hypothetical missing studies were imputed and the pooled analysis incorporating the hypothetical studies was 3.98\% (95\% CI: 2.63-5.97\%), similar to our results.

Eight studies evaluated risk factors of CTEPH after an acute PE, including a total of 2,716 patients (Table 2). Several patient-specific, CTEPH-specific and radiological factors were proved to be relevant to an increased risk of CTEPH. Of the studies that were included, 6 studies performed a multivariate analysis $(2,5,14-16,21)$, and the study by Guérin et al. and the study by Coquoz et al. just reported unadjusted risk factors $(1,23)$. Previous/recurrent PE or previous VTE $(1,2,14,16)$ and right heart dysfunction at presentation $(1,5,14,21)$ were evaluated as risk factors in more than three studies. The indicators of right heart dysfunction include Initial sPAP, right ventricular (RV) diameter and right ventricle/left ventricle (RV/LV) diameter $>1$. Idiopathic PE $(2,5)$, older age $(1,16)$, and RDW $(14,15)$ were identified as significant risk factors in two studies. Most risk factors were identified in only one study.

\section{Discussion}

Our meta-analysis suggests that CTEPH is not a rare complication after an episode of PE. The overall incidence of CTEPH was $3.13 \%$ (95\% CI: $2.11-4.63 \%$ ). Only one 


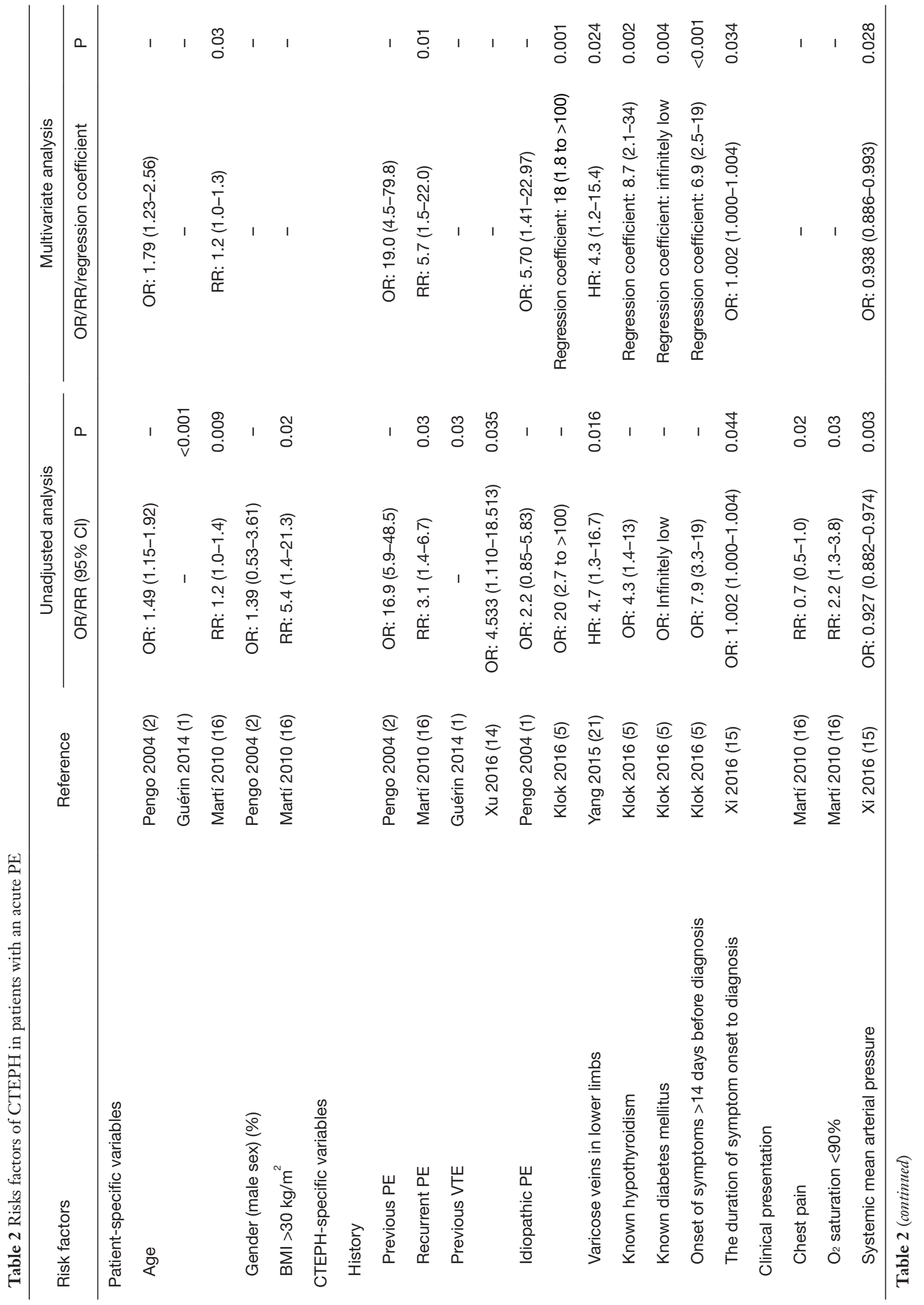




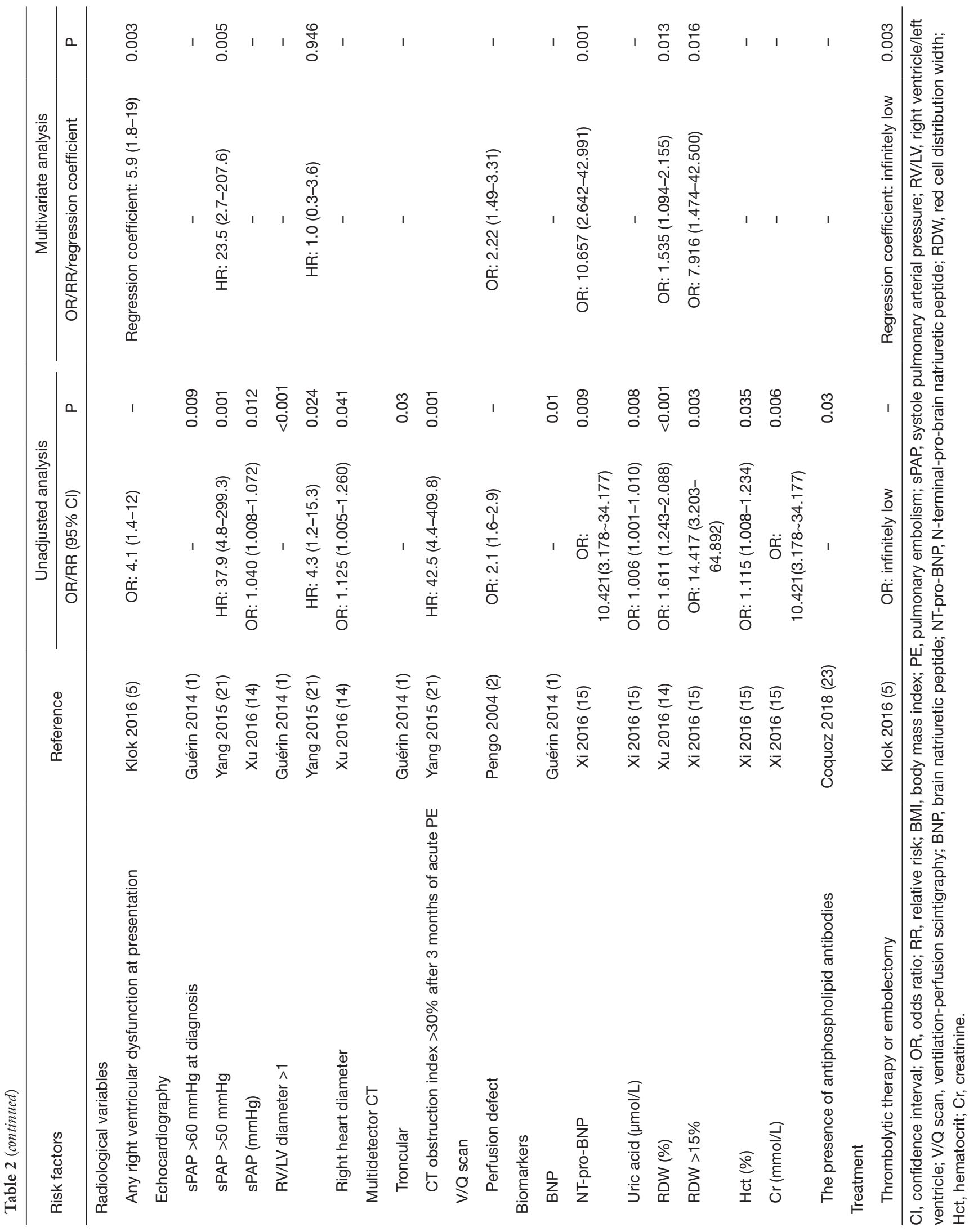


previous systematic review has examined the incidence of CTEPH after an acute PE (24). Compared with the previous meta-analysis $(2.3 \%)$, our results suggested a slightly higher estimate, similarly to the results of the study conducted by Pengo and colleagues (At 2 years: 3.8\%) (2). Several reasons could explain this discrepancy. First, our analysis didn't include abstracts, reviews, and conference proceedings, because it's difficult to assess quality from incomplete data. Second, our analysis included the latest 4 studies published in $2016(5,14,15,22)$ and 1 study published in 2018 (23). Third, the data from China were also included into our meta-analysis $(14,15,21)$. Although our results showed the incidence of CTEPH in Chinese population seems higher than in Europe, no significant difference can be observed between these two groups.

It is not known that using bundled tests that include an echocardiography and V/Q scan/CTPA along with clinical features can accurately diagnose CTEPH in patients not able to undergo right heart catheterization. Our subgroup analyses showed that it is no difference between patients using right heart catheterization and right heart catheterization combined with bundled tests. In Yang et al.'s study, if written consent could not be obtained for the right heart catheterization, the diagnostic criteria of CTEPH was sPAP at rest $>50 \mathrm{mmHg}$ (echocardiography), with the addition of supportive results from V/Q scan and CTPA (21). Considering it is difficult to use right heart catheterization in every suspected patient, bundled tests including echocardiography and V/Q scan/CTPA along with clinical features appear to be helpful in patients unable to perform right heart catheterization. However, caution is warranted in interpretation, and further studies on the standardization of bundled tests are needed for better medicinal utilization.

Most patients were diagnosed within 2 years after an acute PE. Only one patient who developed CTEPH after 4 years of the occurrence of acute $\mathrm{PE}$ was reported in a previous study (3). This tendency might be partly due to the natural process of a thrombus resolution following an acute PE. Residual chronic thrombus was reported in $48 \%$ of patients at the $3 \mathrm{rd}$ month after the first episode of PE, $27.4 \%$ at the 6th month, and $18.2 \%$ at 12 th month. This result highlighted the importance of close monitoring of patients with PE for an early diagnosis of CTEPH (25). Another explanation is that some of the CTEPH patients were misdiagnosed as acute $\mathrm{PE}$, owing to the fact that the first identified clinical presentation of CTEPH may mimic acute PE (1). This finding suggests more frequent surveillance, throughout the first 2 years after being diagnosed with PE, seems reasonable. As the average follow-up period in most studies was within 2 years, the result needs to be interpreted with caution and more studies are required to confirm this hypothesis.

In recent studies, more CTEPH cases with no symptom or few symptoms have been reported $(16,17)$. Clinical silent CTEPH may be easily missed by the present screening strategies deployed. CTEPH is the only cause of pulmonary hypertension that can be cured by pulmonary endarterectomy. Early detection and diagnosis are crucial for the management of CTEPH. Hence asymptomatic patients warrant attention.

Risk factors of CTEPH after an acute PE were also evaluated in our study. In previous studies, several factors were proven to be closely relevant to the development of CTEPH in patients with an acute PE. Some novel risk factors such as plasma $L p(a)$ levels, hyperlipidemia, red cell distribution width, known hypothyroidism and known diabetes mellitus have also been considered to be associated with CTEPH in recent studies $(14,15,26,27)$. In view of limited raw data from present studies, more evidence is needed to lend support to these potential associations. Due to inclusion of only 8 studies into our research combined with insufficient raw data, we were unable to report a common statistic for each of the mentioned potential risk factors.

Previous/recurrent PE or previous VTE and idiopathic $\mathrm{PE}$ are considered to be associated with an increased risk of CTEPH (24). Previous/recurrent PE or previous VTE is commonly believed to be related to the long-term incomplete resolution, which may result in the presence of pulmonary hypertension. Prevention of recurrent VTE would most likely help prevent CTEPH. Adequate anticoagulant treatment would significantly reduce the appearance of recurrent VTE. Although only two studies identified idiopathic $\mathrm{PE}$ as an independent risk factor on multivariate analyses $(2,5)$, other studies also indicated a similar tendency. In the study by Klok et al., they observed a $0.57 \%$ incidence of CTEPH after an acute PE in an unselected large patient series, while a $1.5 \%$ incidence of CTEPH in patients with unprovoked PE (10). Becattini et al. reported an incidence of symptomatic CTEPH of $0.8 \%$, while the incidence of symptomatic CTEPH in patients with idiopathic PE was $1.5 \%$ (19). This is partly because the underlying disease is not treated in patients with idiopathic $\mathrm{PE}$, resulting in continuing thrombus forming. In addition to previous/recurrent PE or previous VTE and idiopathic 
$\mathrm{PE}$, our systematic review indicated that right heart dysfunction at presentation, as reflections of severity of $\mathrm{PE}$, was also associated with the development of CTEPH. However, there is lack of common criteria for clinicians to use these indexes to select potential CTEPH patients.

Although various risk factors have been identified to be associated with CTEPH, it is difficult to apply these risk factors to identify potential CTEPH cases in patients with a previous PE. Klok et al. developed a clinical prediction score to identify high-risk CTEPH in patients with previous cases of PE. The results showed this prediction score identified patients at lower $(0.38 \%)$ or higher $(10 \%)$ risk of CTEPH (5). This prediction score should be validated in other studies, and further investigations should be designed to develop more effective prediction models.

There were several limitations to this systematic review. Firstly, we detected significant heterogeneity across the studies included in incidence analyses. Although potential sources of heterogeneity were further explored using subgroup analyses, we failed to find sources of heterogeneity through subgroup analyses. Sensitivity analyses used by excluding studies one by one and recalculating the pooled data verified the robustness of results. We postulated potential sources of heterogeneity as follows: (I) different constitution of the final study population might play a role in significant heterogeneity among the studies. Several studies analyzed cohorts of selected patients by excluding patients who died irrelevant of CTEPH and patients with permanent risk factors or previous exertional dyspnea. It is more likely to miss potential CTEPH patients if they have other potential causes for pulmonary hypertension; these patients were excluded from some CTEPH studies $(2,16,18)$; (II) different strategies of follow-up may also result in a wide variation of incidences. In several studies, patients who had otherwise unexplained persistent dyspnea on exertion or at rest during follow-up were considered as suspected CTEPH and received further confirming examinations (1-3,13-15,19,23), while other studies included both symptomatic and asymptomatic patients $(5,16-18,20-22)$; (III) the variations in severity of the initial $\mathrm{PE}$ have also been included among the causes of substantial heterogeneity. Based on discussion above, the results need to be interpreted with caution.

Secondly, owing to the paucity of studies and insufficient raw data being reported in the included studies, we didn't pool the original data into a meta-analysis. Most risk factors were identified in only one or two studies, and not all studies conducted rigorous multivariate analyses. Therefore, it is difficult to speculate the predictive ability of these risk factors.

Thirdly, the funnel plot showed the possibility of publication bias. The revised estimate using trim and fill analysis was slightly higher than our results of metaanalysis, suggesting the confidence of the pooled incidence attenuated. Pooled data estimates and results should be interpreted with caution.

Fourthly, NOS was used for the assessment of bias of observational studies, which was not completely applicable in our analysis.

\section{Conclusions}

CTEPH is not a rare complication after acute PE. Close follow-up and implementation of a comprehensive screening program are helpful to select CTEPH patients, especially in patients with the afro mentioned risk factors. Considering it is difficult to use invasive methods in every suspected patient, bundled tests including echocardiography and V/Q scan/CTPA along with clinical features appear to be helpful in patients unable to perform right heart catheterization. Future researches designed to explore other potential risk factors and the use comprehensive statistical adjustments for potential confounders are required.

\section{Acknowledgements}

Funding: This study was supported by a grant of the China Key Research Projects of the 12th National Five-Year Development Plan (2012BA105B00, 2013BA109B00) and the fund of National Natural Science Foundation of China (31670928).

\section{Footnote}

Conflicts of Interest: The authors have no conflicts of interest to declare.

\section{References}

1. Guérin L, Couturaud F, Parent F, et al. Prevalence of chronic thromboembolic pulmonary hypertension after acute pulmonary embolism. Thromb Haemost 2014;112:598-605.

2. Pengo V, Lensing AW, Prins $M H$, et al. Incidence of chronic thromboembolic pulmonary hypertension after pulmonary embolism. N Engl J Med 2004;350:2257-64. 
3. Poli D, Grifoni E, Antonucci E, et al. Incidence of recurrent venous thromboembolism and of chronic thromboembolic pulmonary hypertension in patients after a first episode of pulmonary embolism. J Thromb Thrombolysis 2010;30:294-9.

4. Mi J, Sun Z, Zhong M, et al. Predictive factors of chronic thromboembolic pulmonary hypertension in patients with acute pulmonary thromboembolism. Zhonghua Xin Xue Guan Bing Za Zhi 2012;40:497-501.

5. Klok FA, Dzikowska-Diduch O, Kostrubiec M, et al. Derivation of a clinical prediction score for chronic thromboembolic pulmonary hypertension after acute pulmonary embolism. J Thromb Haemost 2016;14:121-8.

6. Moher D, Liberati A, Tetzlaff J, et al. Preferred reporting items for systematic reviews and meta-analyses: the PRISMA statement. BMJ 2009;339:b2535.

7. Wells GA, Shea B, O'Connell D, et al. The NewcastleOttawa Scale (NOS) for assessing the quality of nonrandomised studies in meta-analyses. Available online: http://www.ohri.ca/programs/clinical_epidemiology/ oxford.asp

8. Dutt TS, Mohan BV, Tousheed SZ, et al. Incidence of chronic thrombo-embolic pulmonary hypertension following acute pulmonary thrombo-embolism: an Indian perspective. Indian J Chest Dis Allied Sci 2013;55:205-7.

9. Berghaus TM, Barac M, von Scheidt W, et al. Echocardiographic evaluation for pulmonary hypertension after recurrent pulmonary embolism. Thromb Res 2011;128:e144-7.

10. Klok FA, van Kralingen KW, van Dijk AP, et al. Prospective cardiopulmonary screening program to detect chronic thromboembolic pulmonary hypertension in patients after acute pulmonary embolism. Haematologica 2010;95:970-5.

11. Klok FA, Tesche C, Rappold L, et al. External validation of a simple non-invasive algorithm to rule out chronic thromboembolic pulmonary hypertension after acute pulmonary embolism. Thromb Res 2015;135:796-801.

12. Surie S, Gibson NS, Bresser P, et al. Is screening for chronic thromboembolic pulmonary hypertension in patients with a previous pulmonary embolism indicated? Am J Respir Crit Care Med 2009;179:A3330

13. Surie S, Gibson NS, Gerdes VE, et al. Active search for chronic thromboembolic pulmonary hypertension does not appear indicated after acute pulmonary embolism. Thromb Res 2010;125:e202-5.

14. Xu W, Cui J, Ma H, et al. Investigation of prognostic effect of red cell distribution width on chronic thromboembolic pulmonary hypertension in patients with acute pulmonary embolism. Chin J Geriatr 2016;35:581-6.

15. Xi Q, Wang Y, Liu Z, et al. Effect of Red Cell Distribution Width on Long-term Follow-up Study in Patients With Acute Pulmonary Thromboembolism. Chin Circ 2016;31:65-8.

16. Martí D, Gómez V, Escobar C, et al. Incidence of symptomatic and asymptomatic chronic thromboembolic pulmonary hypertension. Arch Bronconeumol 2010;46:628-33.

17. Kayaalp I, Varol Y, Cimen P, et al. The incidence of chronic thromboembolic pulmonary hypertension secondary to acute pulmonary thromboembolism. Tuberk Toraks 2014;62:199-206.

18. Giuliani L, Piccinino C, D'Armini MA, et al. Prevalence of undiagnosed chronic thromboembolic pulmonary hypertension after pulmonary embolism. Blood Coagul Fibrinolysis 2014;25:649-53.

19. Becattini C, Agnelli G, Pesavento R, et al. Incidence of chronic thromboembolic pulmonary hypertension after a first episode of pulmonary embolism. Chest 2006;130:172-5.

20. Miniati M, Monti S, Bottai M, et al. Survival and restoration of pulmonary perfusion in a long-term followup of patients after acute pulmonary embolism. Medicine 2006;85:253-62.

21. Yang S, Yang Y, Zhai Z, et al. Incidence and risk factors of chronic thromboembolic pulmonary hypertension in patients after acute pulmonary embolism.J Thorac Dis 2015;7:1927-38.

22. Vavera Z, Vojacek J, Pudil R, et al. Chronic thromboembolic pulmonary hypertension after the first episode of pulmonary embolism? How often? Biomed Pap Med Fac Univ Palacky Olomouc Czech Repub 2016;160:125-9.

23. Coquoz N, Weilenmann D, Stolz D, et al. Multicentre observational screening survey for the detection of CTEPH following pulmonary embolism. Eur Respir J 2018;51.

24. Ende-Verhaar YM, Cannegieter SC, Vonk Noordegraaf A, et al. Incidence of chronic thromboembolic pulmonary hypertension after acute pulmonary embolism: a contemporary view of the published literature. Eur Respir J 2017;49.

25. Korkmaz A, Ozlu T, Ozsu S, et al. Long-term outcomes in acute pulmonary thromboembolism: the incidence of chronic thromboembolic pulmonary hypertension and associated risk factors. Clin Appl Thromb Hemost 
2012;18:281-8.

26. Gary T, Starz I, Belaj K, et al. Hyperlipidemia is associated with a higher PAP in patients after pulmonary embolism.

Hamostaseologie 2011;31:A5.

Cite this article as: Zhang M, Wang N, Zhai Z, Zhang M, Zhou R, Liu Y, Yang Y. Incidence and risk factors of chronic thromboembolic pulmonary hypertension after acute pulmonary embolism: a systematic review and meta-analysis of cohort studies. J Thorac Dis 2018;10(8):4751-4763. doi: 10.21037/jtd.2018.07.106
27. Ignatescu M, Kostner K, Zorn G, et al. Plasma Lp(a) levels are increased in patients with chronic thromboembolic pulmonary hypertension. Thromb Haemost 1998;80:231-2. 